(C)2009 IEEE. Personal use of this material is permitted. However, permission to reprint/republish this material for advertising or promotional purposes or for creating new collective works for resale or redistribution to servers or lists, or to reuse any copyrighted component of this work in other works must be obtained from the IEEE. 


\title{
User Contribution Measurement Model for Web-based Discussion Forums
}

\author{
Kevin Chai, Vidyasagar Potdar and Elizabeth Chang \\ Digital Ecosystems and Business Intelligence (DEBI) Institute \\ Curtin University of Technology, Perth, Australia \\ e-mail: kevin.chai@postgrad.curtin.edu.au, (v.potdar,e.chang)@curtin.edu.au
}

\begin{abstract}
The success of social software depends on contributions made by two key entities; the infrastructure provider(s) and the content providers (users). Currently, social software providers do not possess a powerful and generic approach to measure the contributions of their users. The ability of measuring user contributions will allow social software providers to accurately identify, acknowledge and reward their content contributors. As a result, content providers may become motivated to contribute content more regularly. This paper proposes a user contribution measurement model which is validated against an operational web-based discussion forum.
\end{abstract}

Index Terms-social software, discussion forums, user contribution measurement, user motivation.

\section{INTRODUCTION}

Social software can be simply defined as, "software that supports group interaction" [1]. Social software comprises of communication and interaction tools such as instant messaging programs, chat programs, discussion forums, blogs, wikis and collaborative real-time editors.

The success of social software depends on the contributions made by two key entities; the infrastructure provider(s) and the content providers (users). The infrastructure provider supplies the platform (e.g. the website) and the content providers contribute the content (e.g. text, pictures and videos). This content is referred to as User Generated Content (UGC) [5, 6].

There has been continuing interest in the adoption of social software in recent years. A number of social software articles have reported that there are at least 174 social networking websites available on the Web today and the majority of these websites are experiencing continuing growth $[15,16,22]$. However, the actual number of social software websites inclusive of forums, blogs and wikis would range in to the millions when one considers the number of opensource social software platforms freely available and abundance of domain specific online communities.

Popular social software websites such as Facebook, MySpace and YouTube have been offered and/or sold for hundreds of millions to billions of dollars $[3,12,18]$ which has attracted many entrepreneurs to its industry. It will be interesting to see whether there will be increases to the number of new social software websites and its growing adoption rate in the near future.

The focus of this research is to develop an automated User Contribution Measurement (UCM) model for webbased discussion forums. The significance of this model is to provide a tool for forum providers in accurately identifying, acknowledging and rewarding users for their contribution and could possibly be applied to distance education and revenue sharing.

The paper is structured as follows. Section 2 evaluates existing literature in the realm UCM models and web-based discussion forums. Section 3 proposes the conceptual model and describes the UCM calculation approach. Section 4 details the experimental settings. Section 5 presents a discussion of the experimental results generated from validating the UCM prototype against an online gaming community. Section 6 concludes the paper with future directions.

\section{LITERATURE REVIEW}

Extensive research has been conducted to understand why people contribute to online communities $[19,20,25$, 26]. [28] defined and tested an expectancy-theory framework that implemented a set of reinforcement constructs to identify how extrinsic incentives (i.e. gifts, social recognition and feedback) could alter a person's performance and contribution. The ability to measure and apply reinforcement constructs was referred to as feedback.

In order for social software providers to achieve feedback, they must decide upon and implement a model to measure user contribution. A developed model could evaluate factors relating to the quantity and quality of UGC. When tactfully implemented, reinforcement can strengthen contributors existing attributes of self-efficacy, intrinsic motivation, desire to achieve and trust [25]. This can result in the production of more UGC and increased user participation. Related research has also suggested that displaying a user's contribution information and/or comparative information of how users are ranked within a community, can motivate them to contribute further $[7,8,11,14,20]$.

Research conducted by [20] on a social movie review website, MovieLens, provided evidence that users were more motivated to rate specific movies that were shown to be more beneficial to the community when compared to movies that were displayed as being less beneficial. For example, if a particular movie has not been rated by many users then a new rating of that movie would be more beneficial compared to a movie that has already been rated by many users. A UCM model could later be tailored to exploit this relationship by displaying the predicted user contribution scores for different types of system activities to encourage certain types of contribution activities. 
A study conducted on featured articles within the German Wikipedia website proposes that "it matters who contributes", when it uncovered a positive correlation between reputable article authors and high quality featured articles [24]. A UCM model that can identify and acknowledge these excellent contributors could result in further high quality UGC. Amazon.com is an example of a website that implements an undisclosed UCM to determine and provide recognition for their top 10 and 50 product reviewers [2].

\section{A. Existing UCM Models}

[7] devised a strategy to motivate user contributions in their peer-to-peer (P2P) community, Comtella, by introducing hierarchical membership levels (gold, silver and bronze). This motivational strategy is mainly based on three social psychology theories which include the reciprocation theory, social validation and the theories of discrete emotions (fear). These membership levels were assigned to users based on their weekly contributions. Users with higherlevel memberships were granted better services and special P2P privileges.

[7] identified that they needed a model for accurately measuring user contributions to implement this incentive mechanism. This notion is supported by previous work by [17] in proposing a need for a contribution-reward mechanism and individual profile generation to display user contributions to motivate additional contribution.

The model developed by [7] included the evaluation of five contribution relevant factors. The results from their model were displayed to users and updated weekly. It was observed from their experimental results that user contributions increased but the quality of contributions declined. Their results highlighted the need for anti-fraud mechanisms in a UCM model to detect and deal with users who fraudulently generate unfair contribution scores.

[8] identified that it was important to control the amount of user contributions, motivate users to contribute highquality resources and repress the contribution of poorquality resources to achieve a self-maintaining community. Thus, a model to measure the quality of user contributions was required. Without such a model, it was believed that excessive and low-quality contributions could result in information overload which can lead to user withdrawal from using the system [8, 13].

A rating mechanism inspired from the Slashdot reputation management system known as, karma, was introduced to Comtella, which allowed users to rate the quality of UGC. Users with a higher level of membership were able to provide more ratings and therefore be more influential in the community.

A virtual currency named, c-point was also awarded to users based on their membership levels and their reputation of giving high-quality ratings as an incentive to encourage users to provide ratings. These c-points were then used to increase the visibility of a user's content within search results. However, [8] noted that a fair rating mechanism should give all contributions an equal chance to receive ratings at the start to avoid a feedback loop resulting in the
Matthew effect (where the rich get richer).

Similar motivational strategies adopted in Comtella were also implemented in an online firm-hosted forum community. The forum provided a member-controlled point reward system where users who posted questions could allocate points (ranging from $0-10$ ) to users that posted answers based on its quality. Users are placed into a level of membership when they accumulate a certain number of points. The level of seniority / membership of users are displayed as a type of hat which indicates the quality of knowledge contributions made by a user [26].

Social influence and comparison are two social psychology theories that was implemented and tested as mechanisms to encourage contributions in a movie recommender online community named, MovieLens. A UCM model was developed to assess the contributions of users within this community. Users were then sent weekly personalised email newsletters with five contribution related links as well as information of whether they had rated less, equal or more movies than the community median. It was discovered that users who were told they had rated less movies had significantly rated more movies in the week following the newsletter. Interestingly, it was also discovered that males were more motivated to contribute by the social influence and comparison newsletter than women [11].

The use of social influence has also employed in an experiment which involved motivating donations to a public radio station. It was observed that first-time donors who were told about the amount that another member had contributed gave on average $12 \%$ more than first-time donors that were not presented with this information [23]. In another financially related experiment, it was discovered that eBay purchasers were prepared to pay $8 \%$ more for a product if the eBay seller was displayed as having a high reputation than a new seller [21].

Users with high levels of contribution may gain additional utility within the community as they might be viewed as being senior, reputable and trustworthy. Research in studying relationships between users through dialogue has identified a number of trust related factors [4]. Some of these factors could be used to determine the trustworthiness of a user within an online community and their trust value could be used to enhance a User's Contribution Score (UCS). Users may be influenced to vary their contribution levels through social influence and comparison by observing the contributions of similar users [7, 11].

[14] developed a UCM model, which they have termed as an incentive mechanism for an online learning forum community introduced for a project named, Learning Network for Learning Design (LN4LD). Their model evaluates 5 user contribution related factors which include forum postings, replies to posts, post ratings, replies to their posts and post ratings received. While information is not provided, it is assumed that the scores generated from the model were displayed to the forum users. It was identified from their experiment that there was a significant increase of both active and passive (lurking) participation after introducing this incentive mechanism. 
Spymac is an example of a public social software website that adopts revenue sharing through employing what they have term as a complex user contribution ranking algorithm. The algorithm evaluates several features including the user rating, the amount of comments they have receive on their uploaded content, the amount of views they have received on their content and the amount of advertising revenue that the user has help accumulate.

thisisby.us is another website that shares a portion of their advertising revenue with contributing users. The amount of revenue shared for each user is calculated daily through their UCM model to generate what they have called, a user's All-Time Goodness rating, which is calculated by evaluating, $(1+$ content views $) *$ content votes.

\section{B. Web-based Discussion Forums}

Many online communities utilise a range of social software tools to support group interaction. Web-based discussion forums are electronic bulletin boards built on web technologies that allow people to discuss topics of interest. Forums are a mature and popular tool and are of particular interest as they have become increasingly incorporated into many websites. A forum UCM model has been proposed for development as they are widely used, have been in employed in an online environment since the 1970s [27] and many existing web-based discussion forum platforms are believed to already capture user contribution relevant information within their database schema.

Currently, it is also believed that forum platforms provide limiting methods of determining how much forum users have contributed to the forum community. There are many platforms available and each of these platforms capture and display a different set of user contribution related information. Additionally, there are also forum platforms that have been customised to capture and display much richer user contribution related information. Examples of customised forums would include the implementation of a UCM in the Comtella and LN4LD forums mentioned in Section 2.1 in addition to other public forums like the Ubuntu forum. These specific forums have not been extensively reviewed as the proposed UCM has been developed with portability across different forum platforms

User contributions can be derived from a relatively small set of features commonly available amongst many popular web-based discussion forum platforms [10]. These features include but are not limited to the number of posts created by a user, forum membership duration of a user, and a user's membership groups.

These features are often displayed with authorship information of forum posts to allow other users to evaluate the contribution, seniority and credibility of a forum user. This information provides forum users with a means of evaluating the social status of their peers and themselves within an online community. These basic factors can be utilised and extended to develop a more advanced UCM model.

Interestingly, out-of-the-box forum features can usually be extended through the development and use of platform specific add-ons. UCM related add-ons were discovered for two popular forum platforms, phpBB and Simple Machine Forums (SMF) but these were specifically developed for advertisement revenue sharing. While these add-ons provide a revenue sharing solution, they become inappropriate for use in a forum where advertisement revenue is not generated or shared with the community.

Due to the large number of various forum platforms, the UCM model is proposed utilise common features to allow for portability across forum platforms for future development and application. The resulting model should operate independently of any adopted revenue generating or revenue sharing model. Additionally, a developed UCM model would require flexibility so it can be tailored for specific online communities.

\section{Conceptual Model}

This section will present details of the UCM calculation approach, its formula and the features evaluated.

\section{A. UCM Approach}

The proposed UCM model calculates user contributions by assigning users contribution points for each feature. This approach is similar to the models employed by Comtella, LN4LD and thisisby.us $[8,14]$ and provides a simple approach to quantifying feature scores.

For example, if the feature, number of posts created by a user was evaluated, then a user $A$ might receive a score of 60 if they have created 60 posts. This feature can also be normalised against the feature score for all users. i.e. If user $A$ has created 60 posts and there are a total of 600 posts then user $A$ has contributed $10 \%(60 / 600)$ for this feature.

There is also a need to apply a coefficient weighting to each feature as certain features are considered to be more valuable from a contribution point of view than others. As a benchmark, the first feature will be given a coefficient value of 1 and all other features will be assigned a weight that is directly or indirectly based on this.

However, the total of all user scores for each feature can vary over a large numerical range. For example, the total of all UCS for the total number of posts created by all users would probably be much higher than the number of voting polls created by all users as voting polls are generally rarer than posts within forums. Therefore the coefficient value must be derived while also considering the rarity of points for each feature.

If two features are considered as equal (i.e. assigned the same coefficient) without taking in to account its rarity then there may be a large difference of contribution points achieved by these two similar features. This is undesirable as we do not want features to largely skew the overall contribution results.

The number of voting polls created by all users provides excellent comparative example as voting polls are generally rarer than posts within forums. Based on this understanding, a weighting of 4 may be assigned to the number of voting polls created by all users feature due to its rarity when compared against the total number of posts created by all users. 
[7] handled this issue by introducing ceiling values which provides a limit of how much a user can score for a contribution activity before additional contributions of such activity were added with a reduced rate. However, ceiling values have not been included in this UCM model during this first phase of development.

\section{B. UCM Equations}

Let $U=\left\{u_{1}, u_{2}, \ldots, u_{n}\right\}$ be the set of users within a forum community where $n$ represents the total number of users. Let $F_{u}=\left\{f_{1 u}, f_{2 u}, \ldots, f_{m u}\right\}$ be the set of contribution features evaluated for each user $u$ and $W=\left\{w_{1}, w_{2}, \ldots, w_{m}\right\}$ be the set of weighting coefficients assigned to each feature where $m$ represents the total number of features. $U C S_{u}$ is used to denote the contribution score of a user. The formula to calculate the score for each user is presented in equation (1) while the formula to calculate how much a user has contribution as a percentage of all user contributions is presented in equation (2).

$$
\begin{aligned}
U C S_{u} & =\sum_{i=1}^{m} w_{i} f_{i u} \\
U C S_{u} & =\frac{\sum_{i=1}^{m} w_{i} f_{i u}}{\sum_{j}^{u} U C S_{j}}
\end{aligned}
$$

\section{UCM Features}

Initially, 56 UCM features were identified by evaluating existing forum platforms and their database schemas. 16 common features from these platforms were selected for implementation in the development of a prototype. Feature weights were assigned and refined by the forum provider during experimentation. Future work may involve the inclusion or removal features in addition to use of statistics and machine learning to derive these feature weights. The selected features are presented in Table 1.

A sticky topic refers to a topic that a forum moderator has tagged as important or useful so they appear at or near the top of a boards topic list. A naïve approach is adopted for assessing the quality of posts that only includes posts that contain more than 1,000 characters. The frequency of user posts is calculated by dividing the number of posts created by a user with a user's membership duration. This membership duration is measured in months and a minimum floor value of 1 month is applied to avoid newly registered users achieving unfairly high scores relative to older users.

\section{EXPERIMENTAL SETTING}

The UCM prototype was validated against a web-based discussion forum used by a community of online gamers for their Multiplayer Online Role Playing Game (MMORPG) guild. The forum is moderated by one of the authors of this paper and is comprised of 240 users, 228 topics, 15,059 topic views and 711 posts.
Table. 1 - UCM Parameters

\begin{tabular}{|c|l|c|}
\hline Code & Name & Weight \\
\hline F1 & \# of posts created by a user & 1 \\
\hline F2 & \# of voting polls created by a user & 4 \\
\hline F3 & \# of votes cast by a user & 1 \\
\hline F4 & \# of questions asked by a user & 1.5 \\
\hline F5 & \# of questions answered by a user & 2 \\
\hline F6 & \# of topics created by a user & 1.5 \\
\hline F7 & \# of sticky topics created by a user & 4 \\
\hline F8 & $\begin{array}{l}\text { \# of topics that the user has provided } \\
\text { the first reply }\end{array}$ & 2 \\
\hline F9 & \# of responses received user topics & 1.5 \\
\hline F10 & \# of views received for user topics & 0.1 \\
\hline F11 & \# of personal messages sent & 0.1 \\
\hline F12 & \# of personal messages received & 0.2 \\
\hline F13 & \# of topic update notifications & 0.1 \\
\hline F14 & \# of board update notifications & 0.1 \\
\hline F15 & \# of quality posts created & 3 \\
\hline F16 & Frequency of user posts & 3 \\
\hline
\end{tabular}

The result generated from the prototype was evaluated by the forum provider who possessed an intimate knowledge of the forum community and had strong opinions of which users were the highest contributors. Iterations of testing and refinement of the UCM prototype (mainly through ad hoc refinement of feature coefficients) were conducted to align the UCS with the forum provider's judgement. It should be noted that feature coefficients were tuned for this forum community and is likely to require modification in its application to other forum communities.

The generated UCS was analysed in detail to review the top ten contributors for each feature, how the introduction of a new feature effected the cumulative contribution results, the overall scores generated for all forum users, and a comparison of the overall scores generated from individual feature scores. For the scope of this paper, only the overall scores for all forum users and the overall feature scores will be presented. Please note that only the top ten contributors were evaluated as the forum provider initially only provide a list of 7 users which he perceived as top contributors in his forum community.

\section{RESULTS AND DISCUSSION}

The results presented in Table 2, displays the top 10 ranked forum users calculated by the UCM prototype. 7 users were initially predicted to be the most valuable contributors to the forum community by the forum provider. These 7 users comprised of the guild leaders and officers of their gaming community. All 7 of these users secured a position within the top 10 contributors along with 3 other users that were later confirmed as high contributors by the forum provider after viewing the results.

The overall UCM results are displayed in Table 3. It is apparent that certain features generated very high scores while others generated very low scores. This has been largely influenced by the inherent nature of the online gaming forum and ad-hoc determination of feature weightings 
based on the provider's perceived contribution values of the forum community.

Table. 2 - UCM User Results

\begin{tabular}{|c|c|}
\hline User & UCS \\
\hline $\mathrm{A}$ & $488.7(8.91 \%)$ \\
\hline $\mathrm{B}$ & $480.6(8.76 \%)$ \\
\hline $\mathrm{C}$ & $375.9(6.86 \%)$ \\
\hline $\mathrm{D}$ & $351.1(6.40 \%)$ \\
\hline $\mathrm{E}$ & $308.7(5.63 \%)$ \\
\hline $\mathrm{F}$ & $271.1(4.94 \%)$ \\
\hline $\mathrm{G}$ & $181.4(3.31 \%)$ \\
\hline $\mathrm{H}$ & $151.4(2.76 \%)$ \\
\hline $\mathrm{I}$ & $145.6(2.65 \%)$ \\
\hline $\mathrm{J}$ & $136.8(2.49 \%)$ \\
\hline Other 230 users & $2,592.8(47.28 \%)$ \\
\hline Total & $5484.1(100 \%)$ \\
\hline
\end{tabular}

Table. 3 - UCM Feature Results

\begin{tabular}{|c|c|}
\hline Code & UCS \\
\hline F1 & $711(12.96 \%)$ \\
\hline F2 & $4(0.07 \%)$ \\
\hline F3 & $6(0.11 \%)$ \\
\hline F4 & $574.5(10.48 \%)$ \\
\hline F5 & $152(2.77 \%)$ \\
\hline F6 & $342(6.24 \%)$ \\
\hline F7 & $64(1.17 \%)$ \\
\hline F8 & $294(5.36 \%)$ \\
\hline F9 & $721.5(13.16 \%)$ \\
\hline F10 & $1505.9(27.46 \%)$ \\
\hline F11 & $1.4(0.03 \%)$ \\
\hline F12 & $3.2(0.06 \%)$ \\
\hline F13 & $0.2(0 \%)$ \\
\hline F14 & $0(0 \%)$ \\
\hline F15 & $435(7.93 \%)$ \\
\hline F16 & $669.4(12.21 \%)$ \\
\hline Total & $5484.1(100 \%)$ \\
\hline &
\end{tabular}

For example, the experimented forum is used by a group of online gamers who have access to other communication channels such as in-game mail, in-game chat rooms and audio-chat which has reduced the need use the forum to facilitate private conversations or small group conversations.

However, the creation of reference posts that inform other gamers of walkthroughs, strategies and game mechanics are highly valued by this community. Thus a high weighting multiplier has been assigned to the creation of sticky topics feature (F7). Despite having a high weighting multiplier, the corresponding feature (F7) did not score well due to the overall low quantity of sticky topics created.

Another example would be to consider the differences between Slashdot and SeniorNet online communities: the Slashdot community highly values the posting of new items but SeniorNet places much more emphasis in responding to existing posts. Therefore, the weightings and scores for these features will vary across these two online communities. Community judgement can be incorporated to effectively determine feature weightings for specific online communities (P Dell 2007, personal comm., 28 Nov 2007).
The number of views received for user topics (F10) has achieved the highest feature score and is more than double the score of the second highest feature score (F9). F10 scored high in comparison to other features as there were many users who lurked but provide little if any contribution.

It was observed that the forum users who scored particularly well for this feature were the creators of reference posts (F7). The rationale for this is that specific reference posts were regularly referred to by the forum community (certain posts may be updated every second day). The combination of both the number of sticky posts created (F7) and the number of views received for user topics (F10) features has enforced and rewarded what is most valued by this forum community, which is the creation of quality reference posts.

However, the large difference between the scores of the highest scoring feature (F10) and other features is of concern. Although the combined scores of both F7 and F10 align with the perceived values of this forum community, the overall community may suffer as a result of users that only focus on creating reference posts to achieve high contribution scores and not contributing to other areas within the forum community. This rationale can also be applied to other social software platforms. It should be noted that the weighting assigned to F10 was largely reduced to cater for the vast number of topic views. This finding suggests a possible need to introduce ceiling values as implemented by [7, 8] which has been left out in this phase pf development. This mechanism would help encourage users to provide different types of contributions to an online community in order to achieve a high and balanced UCS

The number of posts created by a user (F1) scored the third highest feature and is arguably the most common feature used to derive user contributions within current forum platforms. The creation of forum posts is a fundamental method for users to interact and contribute within a forum community. As previously, [8] identified the need to incorporate the evaluation of content quality in a developed UCM model. This advice has been heeded by introducing the evaluating the number of quality posts created by a user (F15) which resulted in ranking as the fifth highest scoring feature. However, this feature employs a naïve approach to quality assessment (post length) which can be improved in future work.

The development of a generic model to assess the quality of user contributions is challenging task as different communities would naturally have varying definitions for content quality. For example, GroundReport is a social software website that allows users (reporters) to post news stories and could implement a strategy to encourage and reward users to generate quality stories. A definition of a quality story for GroundReport could be stories that posses an average user rating of 4 stars, at least 50 views and have at least 3 content classification tags specified. While this quality definition would be appropriate for GroundReport or other related websites, it is not likely to be appropriate for a gaming community. 
Although the development of the UCM prototype and its validation against a web-based discussion forum was considered a success, it still requires further refinement before it can provide meaningful and accurate results for different forum platforms and online communities.

Another limitation of our research was how our UCM model was tweaked in an ad-hoc and subjective manner. The forum provider was assumed to have a true and correct knowledge of the community values and judgement. Additionally, our model was validated against 7 users that the forum provider predicted to be top forum contributors. It was not feasible for the forum provider to rank all users (240) but a more rigorous validation of contribution may be required for the validation of a UCM on a much larger community consisting of thousands of users.

\section{CONCLUSION}

A UCM model for a web-based discussion forum has been presented in this paper. This model has been developed and validated against an operational web-based discussion forum. The results from this validation have aligned with the forum provider's opinion of the top forum contributors and their perceived values of the forum community.

Future research is planned to further refine this model to include a more advanced content quality assessment framework with spam and fraud tolerant mechanisms in addition to employing various web data mining techniques. More formal experimentation may be required to identify whether this UCM model can provide an accurate measure of contribution from the perspective of the content providers and whether such accuracy is required for specific domains.

\section{REFERENCES}

[1] C. Allen, "Life With Alacrity: Tracing the Evolution of Social Software", 2004, Retrieved April 11, 2007, from http://www.lifewithalacrity.com/2004/10/tracing the evo.html

[2] Amazon Top Reviewers, 2008, Retrieved February 5, 2008, from http://www.amazon.com/gp/customer-reviews/top-reviewers.html

[3] M. Arrington, "YouTube's Magic Number - \$1.5 Billion", 2006, Retrieved January 7, 2008, from

http://www.techcrunch.com/2006/09/21/youtubes-magic-number-15billion/

[4] J. Cassell \& T. Bickmore, "Negotiated Collusion: Modeling Social Language and its Relationship Effects in Intelligent Agents" in Journal of User Modelling and User-Adapted Interaction, vol. 13, no. 3-4, 2003, pp. 89-132.

[5] K. Chai, V. Potdar and E. Chang, "A Survey of Revenue Models for Current Generation Social Software's Systems" in Lecture Notes of Computer Science (LCNS) and Proceedings of the International Conference on Computational Science and Its Applications - ICCSA, 2007, pp. 724-738.

[6] K. Chai, V. Potdar and E. Chang, "A Survey of Revenue Sharing Social Software's Systems" in Proceedings of International, Workshop on Social Interaction and Mundane Technologies (Simtech), 2007.

[7] R. Cheng and J. Vassileva, "User Motivation and Persuasion Strategy for Peer-to-peer Communities" in Proceedings of the 38th Annual Hawaii International Conference on System Sciences (HICSS'05), 2005, pp. 193a.

[8] R. Cheng and J. Vassileva, "Adaptive Reward Mechanism for Sustainable Online Learning Community" in Journal of User Modelling and User-Adapted Interaction, vol. 16, no. 3-4, 2006, pp. 332-336.
[9] M. Degemmis, P. Lops and G. Semeraro, “A content-collaborative recommender that exploits WordNet-based user profiles for neighborhood formation" in Journal of User Modelling and UserAdapted Interaction, vol. 17, no. 3, 2007, pp. 217-255.

[10] J.M. Grohol, "Anonymity and Online Community: Identity Matters", 2006, Retrieved October 29, 2007 from, http://www.alistapart.com/articles/identitymatters

[11] M.F. Harper, S.X. Li, Y. Chen and J.A. Konstan, "Social Comparisons to Motivate Contributions to an Online Community" in Proceedings of The Second International Conference on Persuasive Technology, 2007.

[12] R. Hof, "But \$2 Billion?”, 2007, Retrieved January 7, 2008, from http://www.businessweek.com/the thread/techbeat/archives/2006/03/ facebook for sa.html

[13] P. Kalnis, W.S. Ng, B.C. Ooi and K.L. Tan, "Answering Similarity Queries in Peer-To-Peer Networks" in Journal of Information Systems, vol. 31, no. 1, 2006, pp. 27-72.

[14] R. Klamma, M.A. Chatti, E. Duval, H. Hummel, E.T. Hvannberg, M Kravcik, E. Law, A. Naeve, and P. Scott, "Social Software for Lifelong Learning" in Journal of Educational Technology \& Society, vol. 10, no. 3, 2007, pp. 72-83.

[15] J. Liew, "Top social networks for engagement - some surprises", 2007, Retrieved January 7, 2008, from http://lsvp.wordpress.com/2007/07/23/top-social-networks-forengagement-some-suprises/

[16] A. Lipsman, "comScore: Social Networking Goes Global", 2007, Retrieved January 7, 2008, from http://www.comscore.com/press/release.asp?press $=1555$

[17] S.M. Lui, K.R. Lang and S.H. Kwok, "Participation Incentive Mechanisms in Peer-to-Peer Subscription Systems" in Proceedings of the 35th Hawaii International Conference on System Sciences (HICSS'02), vol. 7, no. 10, 2002, pp. 3925-3931.

[18] M. Marshall, "SiliconBeat: Web 2.0 peak? MySpace sells for $\$ 580$ million", 2005, Retrieved January 7, 2008, from http://www.siliconbeat.com/entries/2005/07/18/web_20_peak myspa ce sells for 580 million.html

[19] T.D. Moore and M.A. Serva, "Understanding member motivation for contributing to different types of virtual communities: a proposed framework" in Proceedings of the 2007 ACM SIGMIS CPR conference on 2007 computer personnel doctoral consortium and research conference: The global information technology workforce, 2007, pp. 153-158.

[20] A.M. Rashid, K. Ling, R.D. Tassone, P. Resnick, P. Kraut and J. Riedl, "Motivating participation by displaying the value of contribution" in Proceedings of the SIGCHI conference on Human Factors in computing systems, 2006, pp. 955-958.

[21] P. Resnick, R. Zeckhauser, J. Swanson and K. Lockwood, "The Value of Reputation on eBay: A Controlled Experiment" in Journal of Experimental Economics, vol. 9, no. 2, 2006, pp. 79-101.

[22] E. Schonfeld, "Social Site Rankings (October, 2007)", 2007, Retrieved January 7, 2008, from http://www.techcrunch.com/2007/11/28/social-site-rankings-october$2007 /$

[23] J. Shang and R. Croson, "Field Experiments in Charitable Contribution: The Impact of Social Influence on the Voluntary Provision of Public Goods" to be submitted to Quarterly Journal of Economics.

[24] K. Stein and C. Hess, "Does It Matter Who Contributes? - A Study on Featured Articles in the German Wikipedia" in Proceedings of the 18th conference on Hypertext and Hypermedia, 2007, pp. 171-174.

[25] S.J.J. Tedjamulia, D.L. Dean, D.R. Olsen, C.C. Albrecht, D. Thomas and V. Buch, "Motivating Content Contributions to Online Communities: Toward a More Comprehensive Theory" in Proceedings of the 38th Annual Hawaii International Conference on System Sciences (HICSS), 2005, pp. 193b.

[26] C. Wiertz and K. Ruyter, "Beyond the Call of Duty: Why Customers Contribute to Firm-hosted Commercial Online Communities" in Journal of Organization Studies, vol. 28, no. 3, 2007, pp. 347-376.

[27] D.R. Wooley, "Plato: The Emergence of Online Community" in Computer-Mediated Communication Magazine, vol. 1, no. 3, 1994, pp. 5.

[28] P.M. Wright and K.M. Kacmar, "Mediating roles of self-set goals, goal commitment, self efficacy, and attractiveness in the incentive performance relation" in Journal of Human Performance, vol. 8, no. 4, 1995, pp. 263-296. 